\title{
Neoagarohexaose-mediated activation of dendritic cells via Toll-like receptor 4 leads to stimulation of natural killer cells and enhancement of antitumor immunity
}

\author{
Moon Hee Lee, ${ }^{1, \#}$, Jong-Hwa Jang, ${ }^{2, \#}$, Gun Young Yoon ${ }^{1, \#}$, Seung Jun Lee ${ }^{1, \#}$, Min-Goo Lee ${ }^{3}$, Tae Heung Kang ${ }^{1}$, \\ Hee Dong Han ${ }^{1}$, Hyuk Soon Kim ${ }^{1}$, Wahn Soo Choi ${ }^{1}$, Won Sun Park ${ }^{4}$, Yeong-Min Park ${ }^{1, *}$ E In Duk Jung ${ }^{\text {,* }}$ \\ ${ }^{1}$ Department of Immunology, Laboratory of Dendritic Cell Differentiation and Regulation, School of Medicine, Konkuk University, \\ Chungju 27478, ${ }^{2}$ Department of Dental Hygiene, Hanseo University, Seosan $31962,{ }^{3}$ Department of Physiology, Korea University, College \\ of Medicine, Seoul 02841, ${ }^{4}$ Institute of Medical Sciences, Department of Physiology, Kangwon National University School of Medicine, \\ Chuncheon 24341, Korea
}

\begin{abstract}
$\beta$-Agarase cleaves the $\beta-1,4$ linkages of agar to produce neoagarooligosaccharides (NAO), which are associated with various physiological functions. However, the immunological functions of NAO are still unclear. In this study, we demonstrated that $\beta$-agarase DagA-produced neoagarohexaose (DP6), an NAO product, promoted the maturation of dendritic cells (DCs) by Toll-like receptor 4 (TLR4). DP6 directly and indirectly enhanced the activation of natural killer (NK) cells in a TLR4-dependent manner in vitro and in vivo. Finally, the antitumor activity of DP6 against B16F1 melanoma cells was inhibited in NK cell-depletion systems by using NK-cell depleting antibodies in vivo. Collectively, the results indicated that DP6 augments antitumor immunity against B16F1 melanoma cells via the activation of DC-mediated NK cells in a TLR4-dependent manner. Thus, DP6 is a potential candidate adjuvant that acts as an immune cell modulator for the treatment of melanoma. [BMB Reports 2017; 50(5): 263-268]
\end{abstract}

\section{INTRODUCTION}

Agar, composed of $\beta$-D-galactose linked by $\beta-1,4$ linkages and 3,6-anhydro- $\alpha$-L-galactose linked by $\alpha-1,3$ linkages, can be hydrolyzed by $\alpha$-agarase and $\beta$-agarase, respectively (1).

*Corresponding authors. In Duk Jung, Tel: +82-2-2049-6330; Fax: +82-2-2049-6192; E-mail: jungid@kku.ac.kr, Yeong-Min Park, Tel: +82-2-2049-6158; Fax: +82-2-2049-6192; E-mail: immun3023@ kku.ac.kr

${ }^{\#}$ These authors contributed equally to this work.

https://doi.org/10.5483/BMBRep.2017.50.5.014

Received 8 February 2017, Revised 2 March 2017, Accepted 9 March 2017

Keywords: Antitumor immunity, Dendritic cells, Natural killer cells, Neoagarohexaose, Toll-like receptor 4 $\beta$-agarase cleaves the $\beta-1,4$ linkages of agar, producing neoagarooligosaccharides (NAO) including neoagarobiose (DP2), neoagarotetraose (DP4), and neoagarohexaose (DP6) (2). Several reports suggest that NAO inhibit bacterial growth (3), and stimulate macrophages (4); however, the functional analysis of DP2, DP4, or DP6, which are single compounds of $\mathrm{NAO}$, has not been undertaken. In this study, we used $\beta$-agarase (DagA) purified from Streptomyces coelicolor A3(2), a non-toxic, gram-positive soil bacterium that is well known for its use in the production of approximately $85 \%$ of all antibiotics (1). However, the immunological functions of $\beta$-agarase DagA-produced NAO, especially DP6, remain unclear. Therefore, we focused on the physiological functions of $\beta$-agarase DagA-produced DP6, especially, its role in the activation of dendritic cell (DC)-mediated cancer immunotherapy to verify the potential of DP6 as an immunomodulatory agent.

The communication between NK cells and DCs influences both innate and adaptive immunity and enhances Th1 and CTL-mediated antitumor efficacy (5). Mature DCs (MHC II $\left.{ }^{\text {high }} \mathrm{CD} 86^{\text {high }} \mathrm{CD} 11 \mathrm{C}^{+}\right)$stimulate NK cells via soluble factors (IL-2, IL-12, IL-15, IL-18, IFN- $\alpha$, and IFN- $\beta$ ), as well as direct cell-to-cell contact (ligation of NKp46, NKp30, NKG2D, 2B4, and CD27, as well as IL-15 in trans), leading to cytotoxicity, cytokine secretion (IFN- $\gamma$ and TNF- $\alpha$ ), and proliferation of NK cells (11). In contrast, IFN- $\gamma$-producing NK cells $\left(\mathrm{CD} 69^{+} \mathrm{NK} 1.1^{+}\right)$ induce the maturation of DCs and type- 1 polarized DCs producing pro-inflammatory cytokines (6). In addition, NK cell-derived IFN- $\gamma$ up-regulates Th1 transcription factor GATA-3 (6). The interaction between NK cells and DCs reportedly regulates NK and T-cell responses against target cells (7).

In this study, we aimed to identify the immunological actions of the natural polysaccharide DP6. DP6 activates DCs by activating mitogen-activated protein kinases (MAPKs) and nuclear factor- $\mathrm{kB}(\mathrm{NF}-\kappa \mathrm{B})$ signaling via Toll-like receptor 4 (TLR4). In addition, the administration of DP6 showed 
TLR4-dependent antitumor effects against B16F1 melanoma in vivo. Further, stimulation of NK cells in response to DP6 was demonstrated in vitro and in vivo. In addition, DP6-induced maturation of DCs was shown to trigger stimulation of NK1.1 $1^{+}$ cells in vitro. Lastly, NK cell-mediated antitumor immunity in response to DP6 was investigated in vivo. Collectively, the results from this study provide new insights into the immunomodulatory activities of DP6. To our knowledge, this is the first study to demonstrate that DP6 induces DC maturation, leading to the stimulation of NK cells and the enhancement of antitumor immunity.

\section{RESULTS}

\section{DP6 induces the activation of DCs via TLR4}

To verify the products of agar hydrolysis, reaction products including DP6 were separated and identified by thin layer chromatography (TLC). As shown in Supplementary Fig. 1A, the purified single compounds, DP6 (M1), were observed on a TLC plate, as compared to DagA-derived NAO, which was used as a positive marker (M2). The purity of DP6 after gel permeation chromatography was quantified by HPLC Evaporative Light Scattering Detector (HPLC ELSD) as approximately 95\% (Supplementary Fig. 1B). To exclude the possibility of contamination with endotoxin, the purified DP6 was subjected to LAL endotoxin detection assay (Supplementary Fig. 1C) and silver staining (Supplementary Fig. 2), followed by confirmation that the purified DP6 sample was not contaminated with endotoxins. In addition, to evaluate the cytotoxic effects of DP6 on dendritic cells (DCs), the rate of apoptosis or necrosis in DP6-treated DCs was analyzed by flow cytometry (Supplementary Fig. 1D). While treatment with $\mathrm{H}_{2} \mathrm{O}_{2}$, as an apoptosis inducer, markedly increased the apoptosis, DP6 had no cytotoxic effects in DCs.

To investigate whether DP6 affects the maturation of DCs, we measured the expression of surface molecules (CD80, CD86, MHC class I, and MHC class II). The surface molecules showed increased levels of expression in DP6-treated DCs as well as LPS-treated DCs (Fig. 1A-left and right panels, respectively). In addition, the secretion of pro- and antiinflammatory cytokines (TNF- $\alpha$, IL-6, IL-1 $\beta$, IL-12p70, and IL-10) was significantly elevated in DP6-treated DCs (Fig. 1B). Moreover, DP6 significantly diminished the percentage of dextran ${ }^{+} \mathrm{CD} 11 \mathrm{c}^{+}$cells (Fig. 1C), as compared to untreated DCs, indicating that DP6 enhances phenotypic and functional maturation of DCs.

Toll-like receptors (TLRs) are considered to play an important role in the activation of DCs (8); and TLR4 is necessary for the activation of immune cells by several natural polysaccharides (9). Therefore, to examine whether TLR signaling is involved in DP6-mediated DC activation, the expression of surface molecules and the production of cytokines were measured in DP6-treated DCs derived from $\mathrm{WT}, \mathrm{TLR}^{-1-}, \mathrm{TLR}^{-1-}$, and $\mathrm{TLR}^{-1-}$ mice. In DCs from
$\mathrm{TLR}^{-/-}$mice, DP6 induced the expression of surface molecules and significantly decreased the production of cytokines, as compared to DCs from WT, TLR2 ${ }^{-1-}$, and $\mathrm{TLR9}^{-1-}$ mice (Fig. 2A and 2B).

Next, to investigate whether DP6 stimulates the activation of MAPKs, AKT, and NF- $\kappa \mathrm{B}$, which are crucial for TLR4-mediated DC activation (10), the phosphorylation levels of MAPKs and AKT and the degradation levels of p65 in response to DP6 were identified in DCs from WT and TLR4 ${ }^{-1-}$ mice (Fig. 2C). As shown in Fig. 2C, DP6 induced phosphorylation of ERK,

A
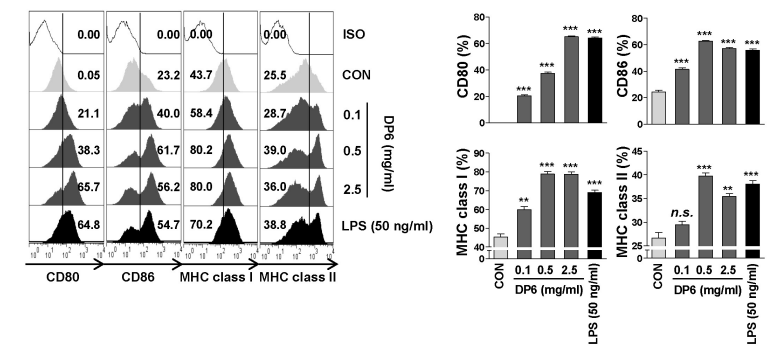

B

C

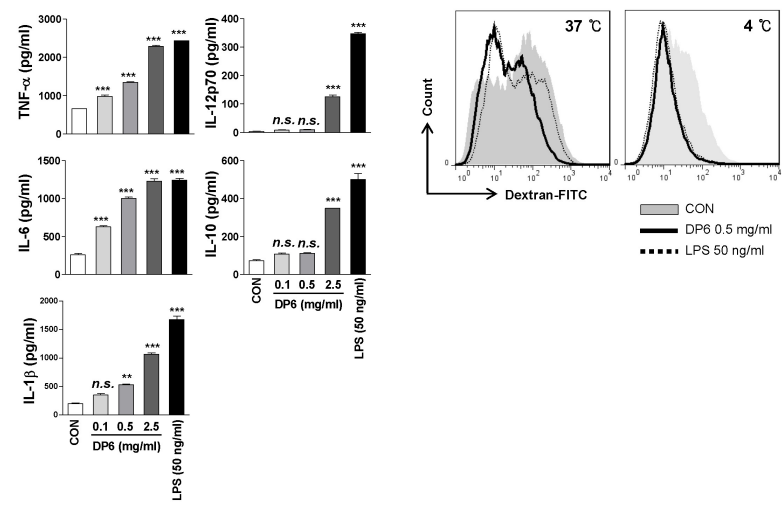

Fig. 1. DP6 induces the phenotypical and functional maturation of DCs. (A, B) Immature DCs were treated with $0.1,0.5$, and 2.5 $\mathrm{mg} / \mathrm{ml}$ DP6 or $50 \mathrm{ng} / \mathrm{ml}$ LPS for $24 \mathrm{~h}$. (A) Flow cytometry was used to analyze the expression of surface molecules on $\mathrm{CD}_{11 \mathrm{c}}{ }^{+}$ cells (left panel). The percentage of positive cells is shown in each panel and bar graph (right panel). The results of one representative experiment out of three experiments are shown. The data are presented as the means and standard error of the mean (SEM). ${ }^{* * P}<0.01$ and $* * * P<0.001$ compared to untreated DCs. (B) ELISA was performed to measure the TNF- $\alpha$, IL-6, IL-1 $\beta$, IL-12p70, and IL-10 production in DP6- or LPS-treated DCs. The data are presented as the means and standard error of the mean (SEM, $n=3$ ). **P $<0.01$ and $* * * P<0.001$ compared to untreated DCs. (C) Endocytic activity of DP6-treated DCs. Endocytic activity of dextran-FITC uptake by DCs treated with medium, LPS, or DP6 was assessed at $37^{\circ} \mathrm{C}$ or $4^{\circ} \mathrm{C}$ (as a control) by flow cytometry analysis. The percentages of dextran-CD11c ${ }^{+}$ cells are indicated. The results of one representative experiment out of three experiments with similar results are shown. 
A

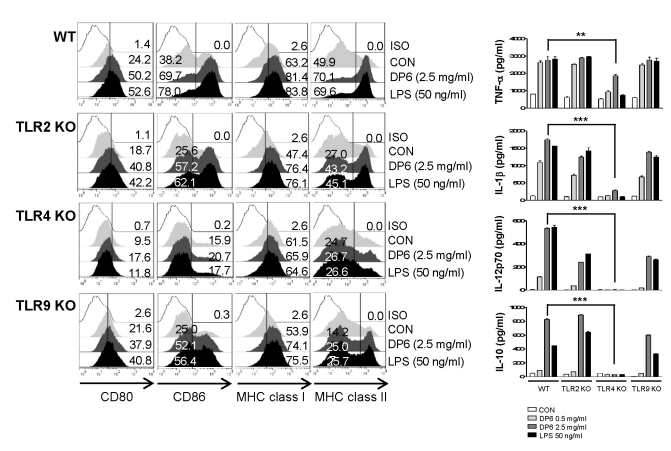

C

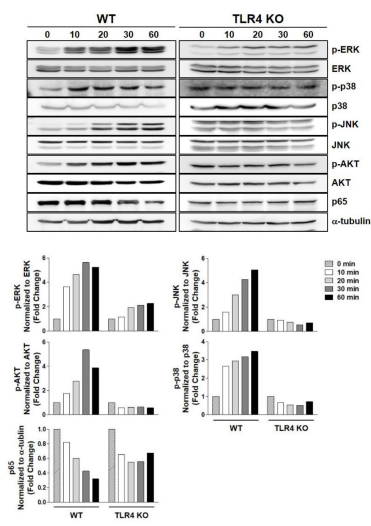

Fig. 2. DP6 induces Toll-like receptor 4 (TLR4)-mediated DC activation. (A, B) Immature DCs from WT, TLR2 $2^{-1-}$, $\mathrm{TLR}^{-1-}$, and TLR9 ${ }^{-1-}$ mice were treated with 0.5 or $2.5 \mathrm{mg} / \mathrm{ml}$ DP6 or $50 \mathrm{ng} / \mathrm{ml}$ LPS for $24 \mathrm{~h}$. (A) Histogram showing CD80, CD86, MHC class I, or MHC class II expression on $\mathrm{CD}_{11 \mathrm{C}^{+}}$cells. The percentage of positive cells is shown in each panel. The results of one representative experiment out of three experiments are shown. (B) ELISA was performed to test IL-1 $\beta, \mathrm{IL}-12 \mathrm{p} 70$, and IL-10 production in DP6- or LPS-treated DCs. The data are presented as the means and standard error of the mean (SEM, $n=3$ ). $* * P<0.01$ and $* * * P<0.001$ compared to 2.5 $\mathrm{mg} / \mathrm{ml}$ DP6-treated WT DCs. (C) Immature DCs from WT and TLR4 ${ }^{-1-}$ mice were treated with $1 \mathrm{mg} / \mathrm{ml}$ DP6 at the indicated time points. The cells were harvested, and the cell lysates were detected by immunoblot with anti-p-ERK, anti-ERK, anti-p-p38, anti-p38, anti-p-JNK, anti-JNK, anti-p-JNK, anti-p-AKT, anti-AKT, anti-p65, or anti- $\alpha$-tubulin antibodies (upper panel). The bar graph illustrates the relative intensity of signals from the immunoblots in the upper panel (lower panel).

p38 MAPKs, JNK, and AKT in DCs from WT mice; however, it showed no effect on the phosphorylation of these kinases in DCs from TLR4 ${ }^{-1-}$ mice. In addition, DP6 decreased the level of the p65 subunit of NF-kB in the cytosolic fraction of DCs from WT mice but not in the cytosolic fraction of DCs from $\mathrm{TLR}^{-1-}$ mice. These results indicated that TLR4-mediated activation of MAPKs, AKT, and NF- $\mathrm{kB}$ might be involved in DP6-mediated DC activation.

DP6 augments TLR4-dependent antitumor immunity in vivo Since DCs are known to induce antitumor activity by modulating immune responses (8), the possible antitumor effect of DP6 against B16F1 melanoma cells in vivo was investigated. Briefly, C57BL/6 mice were intraperitoneally (i.p.) administered PBS or DP6 (100 or $200 \mathrm{mg} / \mathrm{kg}$ ), every other day and subcutaneously (s.c.) inoculated with B16F1 melanoma cells during the course of PBS or DP6 administration (Fig. 3A). As shown in Fig. 3B, administration of 100 and $500 \mathrm{mg} / \mathrm{kg}$ DP6 showed significant inhibition of tumor growth compared to that observed in the group administered PBS (Fig. 3B). The therapeutic effect of DP6 against B16F1 melanoma cells was shown in the treated (500 mg/kg DP6) group of mice at 18 days, as compared to the control (PBS) group at the same time-point (Fig. 3C).

TLR4 has a role in DP6-induced DC activation (Fig. 2); hence, the role of TLR4 in DP6-mediated antitumor effects was investigated in WT and TLR4 ${ }^{-l-}$ mice. Mice were i.p. administered $500 \mathrm{mg} / \mathrm{kg}$ DP6 every other day and s.c. inoculated with B16F1 melanoma cells during the course of DP6
A
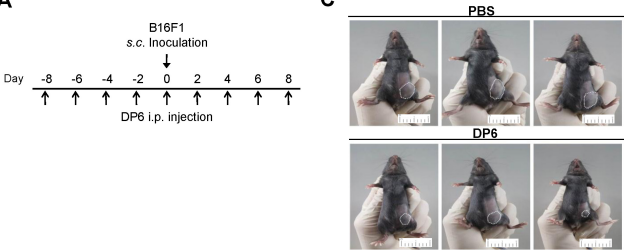

B

D
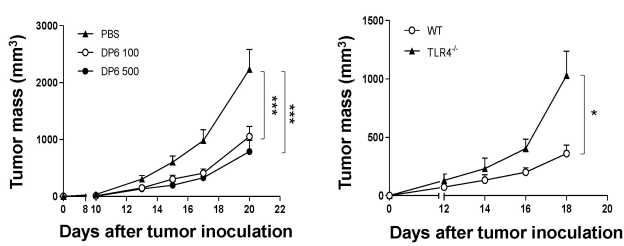

Fig. 3. DP6 augments antitumor immunity in a TLR4-dependent manner in the B16F1 melanoma tumor model. (A-C) Mice (six mice per group) were injected i.p. with PBS or DP6 on days $-8,-6,-4,-2,0,2,4$, and 8 and were injected s.c. with B16F1 melanoma cells. (A) Schematic representation depicting in vivo tumor challenge. (B) Tumor growth was monitored by measuring the diameter of the tumor three times a week. ${ }^{* * * P}<$ 0.001 compared to PBS-injected group. (C) Images of PBS-injected and $500 \mathrm{mg} / \mathrm{ml}$ DP6-injected groups of mice depicting in vivo tumor challenge on day 18. (D) A linear graph summarizing the tumor mass data. WT and TLR4 ${ }^{-l-}$ mice (six mice per group) were injected i.p. with $500 \mathrm{mg} / \mathrm{ml}$ DP6 on days $-8,-6,-4$, $-2,0,2,4$, and 8 and were injected s.c. with B16F1 melanoma cells. Tumor growth was monitored by measuring the diameter of the tumor every other day. Data are expressed as the mean \pm SEM. ${ }^{* P}<0.05$ compared to DP6-injected WT mice. 
administration. Administration of DP6 significantly diminished the tumor mass in WT mice compared to that in TLR4 ${ }^{-1-}$ mice administered DP6 (Fig. 3D). DP6 showed TLR4-dependent antitumor effects on B16F1 melanoma cells in vivo.

\section{DP6 directly and indirectly enhances the activation of NK cells in a TLR4-dependent manner and augments NK cell-mediated antitumor immunity in vitro and in vivo} It has been previously reported that NK cells are essential for killing MHC class I-negative tumor cells such as B16F1 melanoma cells (11). DP6 showed an antitumor effect on B16F1 melanoma cells in vivo in this study (Fig. 3), hence, we evaluated the effect of DP6 on NK cell stimulation in vitro and in vivo. To investigate whether DP6 induces the activation of NK cells in vivo, the activated $\mathrm{CD} 69^{+} \mathrm{NK} 1.1^{+}$cells among the peritoneal exudate cells (PECs) derived from C57BL/6 mice administrated $500 \mathrm{mg} / \mathrm{kg}$ DP6 were identified by flow cytometry analysis. As shown in Fig. 4A, mice administrated DP6 generated a higher percentage of $\mathrm{CD} 69^{+} \mathrm{NK} 1.1^{+}$cells in
PECs as compared to that observed in mice administrated PBS, which was used as a negative control. Next, to verify whether DP6 activates NK cells in vitro, NK1.1 $1^{+}$cells were isolated from C57BL/6 mice by the MACS bead method and then treated with $2.5 \mathrm{mg} / \mathrm{ml}$ of DP6. The percentage of activated CD69 ${ }^{+} \mathrm{NK} 1.1^{+}$cells in response to DP6 was evaluated by flow cytometry analysis (Fig. 4B). The secretions of IFN- $\gamma$ and TNF- $\alpha$ were significantly elevated in culture supernatants from NK cells treated with $2.5 \mathrm{mg} / \mathrm{ml}$ of DP6 (Fig. 4C). Taken together, these results demonstrated that DP6 contributed to NK cell activation in vitro and in vivo.

Since the results indicated that DP6 can activate both DCs (Fig. 1) and NK cells (Fig. 4A-C), we determined whether DP6 controlled the crosstalk between DCs and NK cells, which is a critical factor in anti-cancer immunity (12). To investigate whether DP6 modulates the crosstalk between DCs and NK cells, NK1.1 $1^{+}$cells were treated with the culture supernatant of DP6-treated DCs from WT and TLR4 ${ }^{-1-}$ mice. The percentage of $\mathrm{CD} 69^{+} \mathrm{NK} 1.1^{+}$cells treated with DC supernatant
A
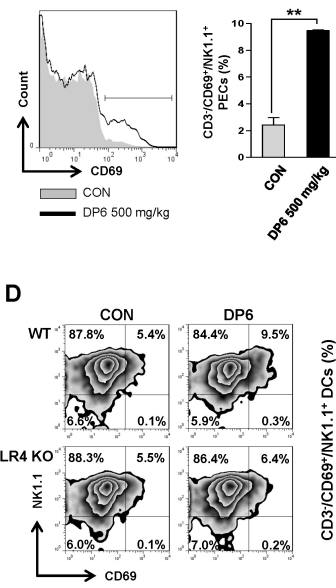

B

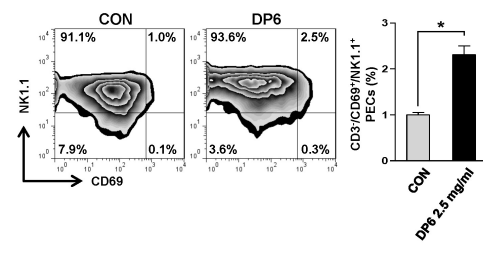

C

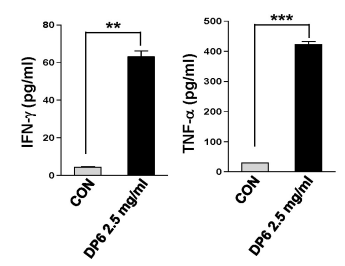

G

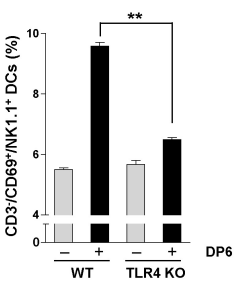

E

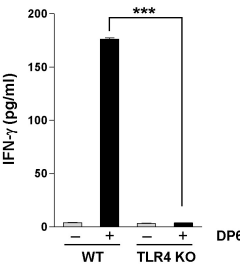

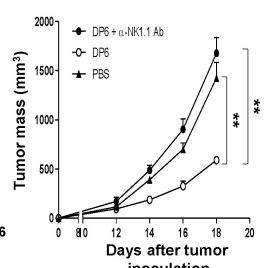

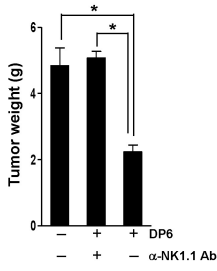

Fig. 4. DP6 directly and indirectly enhances the activation of NK cells in a TLR4-dependent manner and augments NK cell-mediated antitumor immunity in vitro and in vivo. (A) Histogram (left) and bar graph (right) depicting the percentage of CD69 ${ }^{+}$NK1. $^{+}$cells isolated from PECs obtained from PBS- and DP6-injected mice. C57BL/6 mice (five mice per group) were injected i.p. with PBS or DP6. Subsequently, PECs were isolated at $18 \mathrm{~h}$ post-injection and stained and analyzed by flow cytometry analysis. The results of one representative experiment out of three experiments are shown. ${ }^{*} * \mathrm{P}<0.01$ compared to PBS-treated PECs. (B, C) Flow cytometry and ELISA analysis were used to determine the activation of DP6-treated NK1.1 ${ }^{+}$cells in vitro. (B) The percentage of CD69 ${ }^{+}$cells among NK $1.1^{+}$cells was determined by flow cytometry and the data are presented as a histogram (left) and bar graph (right). The results of one representative experiment out of three experiments are shown. ${ }^{*} \mathrm{P}<0.05$ compared to untreated NK cells. (C) ELISA was performed to test IFN- $\gamma$ and TNF- $\alpha$ production in NK1. ${ }^{+}$cells. The data are presented as the means and standard error of the mean (SEM, $n=3$ ). $* * P<0.01 ; * * * P<0.001$ compared to untreated NK1.1 ${ }^{+}$cells. (D, E) Flow cytometry and ELISA analysis were used to determine the activation of $\mathrm{NK}_{1.1^{+}}$CD3 ${ }^{-}$cells treated with the supernatant from PBS- or DP6-treated WT or TLR4 ${ }^{-}$DCs. (D) The percentage of $\mathrm{CD}^{+}{ }^{+}$cells among NK1.1 ${ }^{+}$cells was determined by flow cytometry and the data are presented as a histogram (left) and bar graph (right). The results of one representative experiment out of three experiments are shown. $* * P<0.01$ compared to untreated NK cells. (E) ELISA was performed to measure the IFN- $\gamma$ production in NK1.1 ${ }^{+}$cells. The data are presented as the means and standard error of the mean (SEM, $\mathrm{n}=3$ ). ${ }^{* * * P}<0.001$ compared to NK1.1 ${ }^{+}$cells treated with the supernatant from DP6-treated WT DCs. ( $\mathrm{F}$, G) In vivo antibody depletion experiments. (F) Linear graph depicting the tumor mass of tumor-bearing mice (five mice per group) treated with DP6 with or without NK1.1 depletion. Tumor growth was monitored by measuring the diameter of the tumor every other day. $* * P<0.01$ compared to PBS-injected mice. (G) Bar graph depicting the tumor weight in tumor-bearing mice (five mice per group) treated with DP6 with or without NK1.1 depletion. ${ }^{* P}<0.05$ compared to PBS-injected mice. 
was determined by flow cytometry. Cells cultured with the supernatant from WT-DCs activated by DP6 showed enhanced activation of $\mathrm{CD}^{+} 9^{+} \mathrm{NK} 1.1^{+}$cells and secretion of IFN- $\gamma$; however, these parameters were significantly diminished in cells cultured with the supernatant from TLR4 $4^{-1-}$-DCs activated by DP6 (Fig. 4D and 4E). The data suggested that DP6 causes activation of NK cells by activating DCs via TLR4.

Next, we investigated the role of NK cells in DP6-mediated antitumor effects. The NK cell-depleted mice showed no significant difference in tumor mass and weight as compared to that observed in the group administered PBS (Fig. 4F and 4G). These data demonstrated that NK cells are essential for DP6-mediated antitumor immunotherapy in vivo.

\section{DISCUSSION}

Several natural polysaccharides with immunostimulatory properties have been investigated to date. In addition, natural polysaccharides with immunostimulatory properties such as $\beta$-glucans have been considered as a promising adjuvant to cancer immunotherapy (13). While the immunological functions of $\beta$-agarase-produced NAO have been studied (14), those of NAO produced by nontoxic $\beta$-agarase DagA from Streptomyces coelicolor A3(2) have not been studied yet. In this study, we showed that $\beta$-agarase DagA-produced DP6 1) induced DC activation in a TLR4-dependant manner; 2) triggered the stimulation of NK cells in vitro and in vivo and DP6-activated DCs indirectly induced the stimulation of NK cells in vitro; and 3) induced DC- or NK cell-mediated antitumor immunity via TLR4.

The results of this study demonstrated that DP6 caused the activation of DCs via TLR4 by activating MAPKs, AKT, and NF-KB (Fig. 2C). Our study results suggested that TLR4 acts as the main receptor of DP6 in DC activation, however, other candidate receptors need to be considered because both TLR4 and dectin-1 have been reported as main receptors of $\beta$-glucans, which are well-known natural polysaccharides in DCs (15). Even though dectin-1 is a well-known receptor of polysaccharides in DCs, it is not involved in DP6-mediated DC activation (data not shown). Moreover, it is still unclear whether DP6 might be a ligand of other receptors. Therefore, further studies are required to delineate the involvement of other receptors and related intracellular signaling pathways in response to DP6 treatment.

Since DP6 induced the activation of DCs (Fig. 1), it is possible that DP6 might be effective in cancer immunotherapy. In our experiments, we used the B16F1 melanoma cell line instead of B16F10 for cancer immunotherapy in vivo, because the B16F1 was shown to generate drug-resistant variants at a lower rate than the B16F10 (16). As expected, DP6 administration led to a significant anti-tumor response against B16F1 melanoma cells in vivo. In general, other natural polysaccharides such as $\beta$-glucan do not directly act on cancer cells but enhance antitumor immunity via activation of neutrophils, B cells, T cells, NK cells, dendritic cells, and macrophage-mediated immune responses (17). Since NK cells are essential for the antitumor response against tumors with down-regulated expression of MHC class I molecules such as B16F1 and TC-1 P3 (A15) (18), it is hypothesized that NK cells play a role in DP6-mediated anti-cancer activity against B16F1. The data obtained in this study demonstrated that DP6 enhances NK cell-mediated antitumor immunity, implying that DP6 administration results in antitumor effects against MHC class I down-regulated tumor cells via NK cell-mediated cytotoxicity.

The reciprocal interaction between DCs and NK cells is crucial to enhance the efficacy of anticancer immunotherapy (6). DCs trigger NK cell functions by increasing cytotoxicity, cytokine secretion (IFN- $\gamma, \mathrm{TNF}-\alpha$ ), and proliferation (6). In this study, we demonstrated that DP6 mediated the DC activation; therefore, DP6-activated DCs could potentially trigger the stimulation of NK cells. As expected, soluble factors led to an increase in the percentage of $\mathrm{CD} 69^{+} \mathrm{NK} 1.1^{+}$cells and the level of IFN- $\gamma$ secreted by NK1.1 $1^{+}$cells in vitro, confirming that DP6-activated DCs stimulate NK cells. In future studies, it will be interesting to verify whether DP6-stimulated NK cells activate DCs in reverse.

Overall, our results suggested that DP6 exhibits antitumor activity against B16F1 melanoma cells by controlling the crosstalk between DCs and NK cells via TLR4, indicating that DP6 could be considered a promising adjuvant for cancer immunotherapy.

\section{MATERIALS AND METHODS}

\section{Animal}

Female C57BL/6 mice aged 6-8 weeks were purchased from Orient (Daejeon, Korea) and female C57BL/6J TLR2 knockout mice (TLR2 ${ }^{-I-} ;$ B6.129-TIr $2^{\text {tm1Kir } / J), ~ C 57 B L / 10 ~ T L R 4 ~ k n o c k o u t ~}$ mice (TLR4 $\left.{ }^{-1-} ; \mathrm{C} 57 \mathrm{BL} 10 \mathrm{SCNJ}\right)$, and C57BL/10 TLR9 knockout mice $\left.\left(\mathrm{TLR9}^{-1-} ; \mathrm{C} 57 \mathrm{BL} / 6\right)-\mathrm{Tl}_{\mathrm{r}} \mathrm{M}^{\mathrm{M} B \mathrm{Bt}} / \mathrm{Mmjax}\right)$ were purchased from the Jackson Laboratory (USA) and maintained in filter-top cages under standard conditions (12-h light/dark cycle), with food and water provided ad libitum, in a specific pathogenfree animal facility at the Korea Research Institute of Bioscience and Biotechnology. Animals were sacrificed in the $\mathrm{CO}_{2}$ chamber and organs were harvested instantly.

\section{Cytotoxicity analysis}

Bone marrow-derived dendritic cells (BMDCs) from C57BL/6 mice were treated with $0.5,1.0$, and $2.5 \mathrm{mg} / \mathrm{ml} \mathrm{DP6,} 2.5$ $\mathrm{mg} / \mathrm{ml} \mathrm{LPS}$, and $10 \mathrm{mM} \mathrm{H} \mathrm{H}_{2}$ for $30 \mathrm{~min}$; subsequently, a volume of CellTiter-Glo ${ }^{(\mathbb{2}}$ Reagent (Promega) was added. BMDCs were analyzed for cell viability through luminescence.

\section{Flow cytometry analysis}

The stained cells were analyzed using a FACSCalibur flow cytometer (BD Biosciences, San Jose, CA, USA), and data were 
processed using the Cell Quest Pro software (BD Biosciences).

\section{In vivo Ab-mediated depletion of NK cells}

C57BL/6 (five mice/group) were injected intraperitoneally (i.p.) with the mAb anti-NK1.1 (clone\#:PK136, BioXcell) on days $-2,0,+2,+4,+6,+8,+10$, and +12 (0.2 mg . mouse $^{-1} \cdot d^{-1}$ ) for NK cell depletion. Mice were injected i.p. with $500 \mathrm{mg} / \mathrm{kg}$ DP6 or phosphate-buffered saline (PBS; negative control) on days $2,5,8,11,14$, and 17 . On day 0 , B16F1 melanoma cells $\left(1 \times 10^{5}\right.$ cells/mouse $)$ were injected subcutaneously (s.c.) into the left inguinal region.

\section{Statistical analysis}

All experiments were repeated at least three times. Turkey's multiple comparison tests were used for comparison between samples. Statistical analyses were conducted by using the GraphPad InStat software (Ver 3.1, GraphPad). The data in the graphs are expressed as the mean \pm SEM.

Further information is provided in the Supplementary Methods.

\section{ACKNOWLEDGEMENTS}

This research was supported by the Basic Science Research Program through the National Research Foundation of Korea (NRF) funded by the Ministry of Education, Science and Technology (2013R1A4A1069575, 2014R1A1A2054999, 2015R 1A2A1A13001713, 2016R1D1A3B03934384, and 2016R1A 5A2012284).

\section{CONFLICTS OF INTEREST}

The authors have no conflicting financial interests.

\section{REFERENCES}

1. Temuujin U, Chi WJ, Lee SY et al (2011) Overexpression and biochemical characterization of DagA from Streptomyces coelicolor A3(2): an endo-type beta-agarase producing neoagarotetraose and neoagarohexaose. Appl Microbiol Biotechnol 92, 749-759

2. Fu XT and Kim SM (2010) Agarase: review of major sources, categories, purification method, enzyme characteristics and applications. Mar Drugs 8, 200-218

3. Kono T and Hidaka H (1989) Properties and Production of Neoagarooligosaccharides. J Agric Chem Soc Japan 63, 1126-1129

4. Yoshizawa Y, Ametani A, Tsunehiro J et al (1999) Macrophage stimulation activity of the polysaccharide fraction from a marine alga (Porphyra yezoensis): structure- function relationships and improved solubility. Biosci Biotechnol Biochem 59, 1933-1937

5. Lee SW, Park HJ, Kim N and Hong S (2013) Natural Killer Dendritic Cells Enhance Immune Responses Elicited by alpha-Galactosylceramide-Stimulated Natural Killer T Cells. Biomed Res Int 2013, 460706

6. Van Elssen $\mathrm{CH}$, Oth T, Germeraad WT et al (2014) Natural killer cells: the secret weapon in dendritic cell vaccination strategies. Clin Cancer Res 20, 1095-1103

7. Raulet DH (2004) Interplay of natural killer cells and their receptors with the adaptive immune response. Nat Immunol 5, 996-1002

8. Jung ID, Jeong SK, Lee CM et al (2011) Enhanced efficacy of therapeutic cancer vaccines produced by co-treatment with Mycobacterium tuberculosis heparin-binding hemagglutinin, a novel TLR4 agonist. Cancer Res 71, 28582870

9. Zhang X, Ding R, Zhou Y et al (2013) Toll-like receptor 2 and Toll-like receptor 4-dependent activation of B cells by a polysaccharide from marine fungus Phoma herbarum YS4108. PLoS One 8, e60781

10. Park MJ, Ryu HS, Kim JS et al (2014) Platycodon grandiflorum polysaccharide induces dendritic cell maturation via TLR4 signaling. Food Chem Toxicol 72, 212-220

11. Terao H, Harada M, Kurosawa S et al (1996) The opposite effect of tumor-infiltrating natural killer cells on in vivo priming of tumor-specific CD8 + T cells and CD4 + T cells. Immunobiology 195, 172-186

12. Kalinski P, Nakamura $Y$, Watchmaker $P$ et al (2006) Helper roles of NK and CD8 + T cells in the induction of tumor immunity. Polarized dendritic cells as cancer vaccines. Immunol Res 36, 137-146

13. Aleem E (2013) beta-Glucans and their applications in cancer therapy: focus on human studies. Anticancer Agents Med Chem 13, 709-719

14. Enoki T, Okuda S, Kudo Y et al (2010) Oligosaccharides from agar inhibit pro-inflammatory mediator release by inducing heme oxygenase 1. Biosci Biotechnol Biochem 74, 766-770

15. Karumuthil-Melethil S, Gudi R, Johnson BM et al (2014) Fungal beta-glucan, a Dectin-1 ligand, promotes protection from type 1 diabetes by inducing regulatory innate immune response. J Immunol 193, 3308-3321

16. Cillo C, Dick JE, Ling V and Hill RP (1987) Generation of drug-resistant variants in metastatic B16 mouse melanoma cell lines. Cancer Res 47, 2604-2608

17. Wasser SP (2002) Medicinal mushrooms as a source of antitumor and immunomodulating polysaccharides. Appl Microbiol Biotechnol 60, 258-274

18. Cheng WF, Hung CF, Lin KY et al (2003) CD8 + T cells, NK cells and IFN-gamma are important for control of tumor with downregulated MHC class I expression by DNA vaccination. Gene Ther 10, 1311-1320 